\title{
ENQUETTE ET RECHERCHES RÉCENTES SUR L'INTOXICATION PAR LE FROMAGE DANS LE PROCHE ET MOYEN ORIENT (1)
}

\author{
par \\ AвDo FÉGHALI \\ (Beyrouth, Liban)
}

Ayant récemment observé plusieurs cas d'intoxication chez des personnes ayant consommé diverses variétés de fromage, de fabrication locale, j'ai voulu donner une description détaillée du mécanisme de ces empoisonnements alimentaires, de fréquence inaccoutumée.

Le fromage en Orient, extrait du lait de divers animaux (vache, chèvre, brebis, buffle), comprend plusieurs espèces: yaghourt sec, kariché, labané et fromage vert (Liban), fromage blano ou stambouli (Turquie), fromage jaune ou accaoui (Palestine), fromage en tresse ou halabi (Syrie) et fromage gras ou cachcawân (Grèce, Chypre).

Quoique tous ces fromages soient de différentes provenances, leur mode de fabrication reste le même, ou à peu près. Pour cela, on recueille le lait cru, immédiatement après la traite et tant qu'il est encore tiède ; et, sans le faire bouillir, ni le soumettre à une pasteurisation (Liban, Syrie, Palestine), on y ajoute une "présure spéciale» (2), tout en le remuant doucement, durant deux heures, jusqu'à ce qu'il se sépare en deux substances : une substance solide, qui est le fromage, et une liquide, ou sérum, non utilisée, qu'on rejette.

Aussitôt séparé de son sérum, le fromage est immédiatement saisi par les hommes, femmes et enfants qui, de leurs mains douteuses, en font des galettes rondes, plates, ovales ou tressées ; ils creusent dans chaque morceau une fossette de la profondeur de trois centimètres qu'ils remplissent de sel de cuisine. Deux jours après ce traitement, le fromage est consommable et vendable.

En quoi consiste cette "présuré spéciale»? :

Elle est extraite de l'estomac d'un chevreau nouveau-né qui vient de téter sa mère, et qu'on sacrifiə vingt-quatre heures après sa naissance.

Le liquide de son estomac est conservé dans une vieille outre de peau du même animal, et déposé dans la tente ou dans une cave, pour son utilisation ultérieure.

Beaucoup de tribus et de bergers du désert et des hautes mon-

(1) Presse Médicale, 1952, 22, 487.

(2) Qu "Majbané ». 
tagnes, se servent, en guise de présure, de tout l'estomac de l'animal, qu'ils découpent en petits morceaux, et s'en servent toutes les fois qu'ils désirent préparer du fromage...

Et, quand on pense aux milliards de microbes aérobies et anaérobies, qui peuvent pulluler à l'intérieur de cette "présure spéciale ", ou dans l'outre, autour de l'estomac, non vidé de son contenu, quand on réfléchit à son mode de conservation, ainsi qu'aux mains, éminemment septiques, qui ont pétri le fromage, on ne doit plus s'étonner du nombre impressionnant d'intoxications, parmi la population d'âge scolaire, ainsi que parmi les adultes.

\section{Mécanisme de l'intoxication par le fromage}

Cet empoisonnement provient soit : $a$ ) de l'ustensile même qui a servi à la préparation du fromage; $b$ ) des ptomaïnes ; $c$ ) des herbes consommées par le bétail ; d) des microbes, champignons, bacilles et microorganismes du fromage.

10 L'ustensile. - C'est le cuivre, non étamé ou très mal étamé, qui a servi à la préparation du fromage, qui, par ses sels, sulfures et sulfates, etc., est incriminé dans la majorité des eas d'empoisonnements de ce genre. Les ustensiles faits en zinc, plomb, nickel, pourraient produire, de même, bon nombre d'intoxications.

Afin de prévenir de tels accidents mortels, nous recommandons les ustensiles faits en aluminium, ou, de préférence, en terre cuite émaillée, ou en bois de chêne.

Le mode d'intoxication par les ustensiles en cuivre est très répandu en Orient, depuis l'Antiquité. Il présente une symptomatologie qui ne diffère pas beaucoup de celle de l'arsenicisme, du strychninisme, avec cette même spontanéité, cette même rapidité et ce même tableau qui, au bout de quinze à trente minutes est complet et pourrait même provoquer une mort immédiate, à l'encontre de l'intoxication microbienne qui peut ne pas se déclarer avant dix à vingt-quatre heures, après la consommation de tels fromages toxiques.

$2^{\circ}$ Les ptomaïnes. - Elles existent dans tous les fromages fermentés, et sont constituées par les cadavres des champignons, bacilles, microbes, saprophytes divers du fromage. Ces ptomaïnes ne sont pas, à hotre avis, aussi nocives que certains toxicologues le pensent. Cependant, nous avons observé des signes nets d'intoxication ptomaïnique, quand ces ptomaïnes, se trouvent en abondance dans le fromage. La mort, à la suite de consommation de ce fromage ptomaïné, est rare ; par contre, les troubles digestifs abondent : vomissements, dyspepsie grave et asthénie profonde.

$3^{\circ}$ Les herbes et céréales consommées par le bétail. - On 
accuse populairement, en Orient, les diverses herbes et céréales, de la production des cas d'empoisonnements alimentaires mortels. Scientifiquement parlant, la petite quantité de ptomaïnes toxiques qui passent par le lait, réduit en fromage dans l'estomac du consommateur, est insignifiante. Elle n'arrive pas, logiquement à tuer un homme, sans tuer, au préalable, la bête qui l'a consommée. Cependant, nous avons pu observer des cas de toxicose mortels chez des nourrissons ayant absoré, à certaine saison de l'année, un lait de vache nourrie exclusivement de gesse et de vesce.

$4^{\circ}$ Intoxication mortelle d'origine microbienne. - C'est, sans contredit, la forme la plus fréquente sous nos climats, et partant la plus redoutable.

Des recherches bactériologiques récentes, effectuées en 1951, par nous-même, à l'Hôpital des Enfants-Malades de Beyrouth, avec nos collaborateurs, nous ont permis de déceler le staphylocoque dans $75 \%$ des cas d'intoxication par le fromage. Le staphylocoque arrive à ces fromages par les mains septiques qui les ont moulés, manipulés, ou vendus.

Les personnes ayant des éruptions cutanées, des pyodermites a staphylocoques, ne sont pas rares sous nos climats. De plus, ces fromages, conservés longtemps à une température tiède, favorable par conséquent à la reproduction et à la multiplication du staphylocoque, constituent un excellent réservoir de toxines staphylococciques et un magnifique milieu de culture au staphylocoque luimême.

Symptomatologie. - Uniformément, une personne intoxiquée par les fromages, ainsi que par le lait et les laitages septiques, présente-invariablement : nausée, vomissement, diarrhée, mucus, glaires et pelures d'intestins, coliques et gargouillements continuels.

De plus, il y a malaise, somnolence, photophobie et pseudoparalysie. L'ictère est fréquent ainsi que la néphrite par atteinte du
parenchyme rénal. Il y a albuminurie, ballonnement du ventre, parenchyme rénal. Il y a albuminurie, ballonnement du ventre, fièvre modérée, sécheresse de la peau et des muqueuses, pâleur, facies typhique, asthénie profonde, ou, au contraire, agitation extrême.

Les œdèmes ne sont pas rares, ainsi que l'anurie. La mort survient immanquablement par collapsus (1).

Prophylaxie. - $1^{\circ}$ Déclarer obligatoirement les cas d'intoxica-

(1) Cf. Volume des Comptes Rendus du XIIe Congrès des Pédiatres de Langue française de Paris, ainsi que celui du VIe Congrès International de Pédiatrie de Zürich : Dyspepsie infantile toxique des farines falsifiées et des laitages sepliques, Communications Abdo Féghali. 
tion mortels, et ordonner une enquête serrée dans le quartier, la ville, la commune ou eut lieu l'accident;

20 Réglementer l'industrie des fromages, en accord avec les municipalités et les services de Santé publique ;

$3^{\circ}$ Employer un lait pasteurisé ou faire bouillir convenablement le lait destiné à l'industrie du fromage ;

$4^{\circ}$ Distribuer, sous contrôle chimique, une présure aseptique ;

$5^{\circ}$ Conserver le surplus du fromage frais dans des frigidaires, au même titre que le beurre.

(Clinique médicale infantile de l'Hôpital des Enfants-Malades de Beyrouth.)

\title{
- ASPECTS SCIENTIFIQUES DE LA FABRICATION CONTINUE DU BEURRE (1)
}

\author{
par
}

\section{N. $\mathrm{KING}$}

Section des Recherches laitières. Organisation de Recherches seientifiques et industrielles du Commonwealth, Melbourne (Australie)

Quoiqu'il soit possible de baratter directement le lait, la fabrication pratique du beurre utilise une concentration en deux temps. Dans le premier, qui est l'écrémage, la teneur en matière grasse passe de 3 à $4 \%$ à environ 30 à $40 \%$; dans le deuxième, le barattage et le malaxage, elle augmente de nouveau jusqu'à environ $82 \%$. La préparation de la matière grasse de beurre (butteroil), produit contenant environ $99 \%$ de matière grasse, peut être considérée comme un troisième temps.

En même temps que s'est développée l'industrie beurrière s'est manifestée une tendance accrue à passer du travail intermittent au travail continu. Ce passage, dans le cas de la centrifugation de la crème, s'est produit au cours des dix dernières années du siècle écoulé. Les essais pour placer aussi le second temps de la fabrication du beurre sous le régime de la continuité ("extracteur» de beurre de Johansson, "séparateur " de beurre de de Laval et "radiateur" de beurre de Salenius) eurent lieu assitôt après l'introduction de l'écrémage centrifuge ; mais, malgré cela, n'eurent pas de résultats pratiques. Après un assez long intervalle de temps, ces essais furent repris pendant les trente récentes années du siècle actuel avec le résultat qu'en peu de temps un certain nombre de nouvelles méthodes de fabrication continue du beurre furent perfectionnées,

(1) Dairy Science Abstracts, 1952, 14-4, 226. 